\title{
Nucleos(t)ide Analogs Do Not Independently Influence Hepatic Fibrosis and Portal Hypertension beyond Viral Suppression in CBDL-Induced Cirrhotic Rat
}

\author{
Yu-Hsin Hsieh, ${ }^{1}$ ○Hui-Chun Huang, ${ }^{1}$ Ching-Chih Chang, Chiao-Lin Chuang, Fa-Yauh Lee, \\ (1) Shao-Jung Hsu, Yi-Hsiang Huang, Ming-Chih Hou, and Shou-Dong Lee
}

Division of Gastroenterology and Hepatology, Department of Medicine (Y.-H.Hs, H.-C.H., F.-Y.L., S.-J.H., Y.-H.Hu, M.-C.H.) and Division of General Medicine, Department of Medicine, (Y.-H.Hs, H.-C.H., C.-C.C., C.-L.C.), Taipei Veterans General Hospital, Taipei, Taiwan; Faculty of Medicine, National Yang-Ming University School of Medicine, Taipei, Taiwan (H.-C.H., C.-C.C., C.-L.C., F.-Y.L., S.-J.H., Y.-H.Hu, M.-C.H., S.-D.L.); and Division of Gastroenterology, Department of Medicine, Cheng Hsin General Hospital, Taipei, Taiwan (S.-D.L.)

Received May 26, 2018; accepted August 7, 2018

\begin{abstract}
Chronic hepatitis is the major cause of liver cirrhosis and portal hypertension. Several factors affect portal pressure, including liver fibrosis, splanchnic vasodilatation, and pathologic angiogenesis. Nucleos(t)ide analogs (NUCs), the oral antiviral agents, effectively attenuate chronic hepatitis B-related liver cirrhosis and portal hypertension via viral suppression and alleviation of hepatitis. On the other hand, NUCs affect tumor necrosis factor (TNF)- $\alpha$, vascular endothelial growth factor (VEGF), and nitric oxide, which participate in fibrogenesis, vasodilatation, and angiogenesis. However, whether NUCs independently influence liver fibrosis and portal hypertension beyond viral suppression is unknown. This study thus aimed to evaluate the influences of three frequently used NUCs in rats with nonviral cirrhosis. Male Sprague-Dawley rats received common bile duct ligation (CBDL)
\end{abstract}

to induce cholestatic cirrhosis and portal hypertension. The rats were randomly allocated into four groups, treated by mouth with lamivudine (30 mg/kg per day), entecavir $(0.09 \mathrm{mg} / \mathrm{kg}$ per day), tenofovir (50 mg/kg per day), or distilled water (vehicle control) from the 15th day after CBDL. On the 29th day, liver cirrhosisand portal hypertension-related parameters were evaluated. The results showed that chronic NUCs treatment did not affect hemodynamic parameters, plasma TNF- $\alpha$ concentration, and hepatic fibrogenesis protein expressions in rats with nonviral cirrhosis. Though the mesenteric VEGF receptor 2 phosphorylation was downregulated in NUCs-treated groups, the splanchnic angiogenesis was not influenced. In conclusion, lamivudine, entecavir, and tenofovir had no additional effects on liver cirrhosis and portal hypertension in rats with nonviral cirrhosis.

\section{Introduction}

Repetitive liver injury, including chronic viral hepatitis, results in liver cirrhosis. During the progression of cirrhosis, portal hypertension and relevant complications develop. Portal hypertension is attributed to several factors, including liver fibrosis and enhanced vascular tone (McConnell and Iwakiri, 2018). On the other hand, splanchnic inflow increases because of abnormal vasodilatation and angiogenesis attributed mainly to nitric oxide (NO) and vascular endothelial growth factor (VEGF) (Sumanovski et al., 1999; Mejias et al., 2009). As a result, the blood flow toward the portal system increases, but the outflow is hindered, which leads to elevated portal pressure. To shunt the abnormal blood flow, portosystemic collateral

This study was supported by a grant from Taipei Veterans General Hospital, Taiwan [Grant V106C-077].

${ }^{1}$ Y.-H. Hsieh and H.-C. H. contributed equally to this study.

https://doi.org/10.1124/jpet.118.250431. vessels develop. However, portosystemic collaterals bring lethal complications, such as variceal bleeding and hepatic encephalopathy. Mitigation of portal hypertension is therefore considered pivotal to improving prognosis for the cirrhotic patient. However, the treatment efficacy of pharmacological agents is currently still suboptimal (Solà et al., 2010).

Nucleos(t)ide analogs (NUCs) serve mainly as reverse transcriptase inhibitors that block hepatitis B virus (HBV) from multiplying and therefore reduce viral numbers. Among NUCs, lamivudine is a nucleoside analog of cytidine, entecavir is a deoxyguanosine analog (Sims and Woodland, 2006), and tenofovir is a defective adenosine nucleotide analog (Perry and Simpson, 2009). These drugs effectively suppress the reverse transcription of HBV and attenuate hepatitis. Long-term treatment with these agents even reverses liver cirrhosis caused by chronic hepatitis B (Dienstag et al., 2003; Chang et al., 2010; Marcellin et al., 2013). Theoretically, the resolution of cirrhosis is caused by suppression of viral activity and

ABBREVIATIONS: BW, body weight; CBDL, common bile duct ligation; $\mathrm{Cl}$, cardiac index; eNOS, endothelial NO synthase; Erk, extracellular signalregulated kinases; HBV, hepatitis B virus; HR, heart rate; MAP, mean arterial pressure; MMP, matrix metalloproteinase; NO, nitric oxide; NUCs, nucleos(t)ide analogs; PP, portal pressure; SMA, superior mesenteric artery; TIMP, tissue inhibitor of metalloproteinase; TNF- $\alpha$, tumor necrosis factor- $\alpha$; VEGF, vascular endothelial growth factor; VEGFR2, VEGF receptor 2. 
subsequent amelioration of inflammation. However, recent studies revealed that these NUCs modulate tumor necrosis factor- $\alpha$ (TNF- $\alpha$ ) expression (Zídek et al., 1999; Biswas et al., 2014), NO production (Zídek et al., 2000; Kostecká et al., 2012), and neovascularization (Mizutani et al., 2015), all of which participate in pathogenesis of liver cirrhosis and portal hypertension. If NUCs exert independent benefits in reversing liver fibrosis and portal hypertension, it may be possible to apply them to patients with nonviral chronic liver diseases. However, direct effects of NUCs on cirrhosis and portal hypertension without $\mathrm{HBV}$ infection have not been surveyed.

This study, therefore, aimed to investigate whether the three most prescribed NUCs used to treat chronic hepatitis B ameliorated fibrogenesis and hemodynamic derangements independent of their antiviral activity in rats with nonviral cirrhosis.

\section{Materials and Methods}

Animals. Male Sprague-Dawley rats were used, in which liver cirrhosis was induced by common bile duct ligation (CBDL), as previously described (Cameron and Muzaffar Hasan, 1958). Briefly, 270 - to $300-\mathrm{g}$ rats were anesthetized with $100 \mathrm{mg} / \mathrm{kg}$ ketamine via intramuscular injection and then received a midline abdominal incision. The bile duct was dissected, doubly ligated and transected between the two ligatures. Vitamin $\mathrm{K}$ injections (50 $\mu \mathrm{g} / \mathrm{kg}$ i.m.) were administrated weekly to reduce mortality caused by coagulopathy (Kountouras et al., 1984).

All animal experiments were conducted in agreement with the Institutional Animal Care and Use Committee (IACUC) of Taipei Veterans General Hospital under the approval number 2016-096. All animals were maintained with humanely in accordance with the principles described in the Guide for the Care and Use of Laboratory Animals organized by the U.S. National Academy of Sciences and issued by the National Institutes of Health (NIH Publication No. 86-23, revised 1985).

Experiment Design. To investigate the roles of NUCs in nonviral cirrhosis and portal hypertension, rats were randomly allocated into four treatment groups: 1) control (distilled water, vehicle); 2) lamivudine [30 mg/kg per day, by mouth (PO)]; 3) entecavir $(0.09 \mathrm{mg} / \mathrm{kg}$ per day, PO); 4) tenofovir (50 mg/kg per day, PO). The treatment started on the 15 th day after CBDL surgery. The dosages were determined according to the previous reports suggesting that they exert pharmaceutical effects in rats (Nirogi et al., 2012; Li et al., 2013; Lu et al., 2014). On the 29th day after operations, cirrhosis- and portal hypertension-related parameters were determined.

Measurement of Systemic and Portal Hemodynamics. For the measurement of mean arterial pressure (MAP) and heart rate (Salerno et al., 2014), the right carotid artery was cannulated with a PE-50 catheter. For the assessment of portal pressure (PP), the abdomen was opened with a midline incision, and the mesenteric vein was cannulated with an 18-guage cannula. The catheters were connected to a two-channel recorder (MP45; BIOPAC Systems, Goleta, CA) that converted the input signals into continuous graphs of MAP, heart rate (HR), and PP. Values of MAP, HR, and PP were recorded when the signals achieved stable state (Huang et al., 2012). The superior mesenteric artery (SMA) was identified at its aortic origin and a 5-mm segment was gently dissected free from the surrounding tissue. SMA flow was measured by a pulsed-Doppler flow transducer (TS420; Transonic Systems Incorporated, Ithaca, NY). Hepatic inflow (portal part) was measured by a flow transducer of adequate size. The measurement point was as proximal to the liver as possible.

Thermodilution method was applied to obtain cardiac output, as previously described (Albillos et al., 1992). Briefly, a thermistor was positioned in the aortic arch distal to the aortic valve, and five thermal indicators (100 $\mu \mathrm{l}$ of iced normal saline) was injected sequentially into the right atrium through a PE-50 catheter. A thermodilution curve was developed after each injection. The aortic thermistor was connected to a cardiac output computer Cardiomax III (Columbus Instruments International Co., Columbus, OH). The value of cardiac output was determined as an arithmetic mean of five cardiac output values calculated from thermodilution curves.

Cardiac index [CI; $\mathrm{ml} / \mathrm{min}$ per $100 \mathrm{~g}$ body weight (BW)] was defined as cardiac output per $100 \mathrm{~g}$ BW. Systemic vascular resistance (SVR, mm $\mathrm{Hg} / \mathrm{ml}$ per minute per $100 \mathrm{~g} \mathrm{BW}$ ) was determined as CI divided by MAP. Hepatic vascular resistance (HVR, $\mathrm{mm} \mathrm{Hg} / \mathrm{ml}$ per minute per $100 \mathrm{~g} \mathrm{BW}$ ) was calculated as PP divided by hepatic vascular flow (portal part) per $100 \mathrm{~g}$ BW. SMA resistance (mm Hg/ml per minute per $100 \mathrm{~g} \mathrm{BW}$ ) was determined as (MAP-PP)/SMA flow per $100 \mathrm{~g}$ BW.

Immunofluorescent Study for the Mesenteric Vascular Density. Through CD31 immunofluorescence staining, CD31-labeled microvascular networks in rat mesenteric connective tissue windows were quantified to evaluate the occurrence of mesenteric angiogenesis according to a previous study (Huang et al., 2012). Mesenteric windows (wedge-shaped regions of transparent connective tissue bordered by the intestinal wall and the ileal blood vessel pairs) were dissected, washed in phosphate-buffered saline, and dried on gelatin slides. The mesenteric windows were fixed in methanol at $-20^{\circ} \mathrm{C}$ for 30 minutes and then acetone at $-20^{\circ} \mathrm{C}$ for 10 minutes. Slides were then incubated with mouse anti-rat CD31-biotin (Bio-Rad, Hercules, CA) at $4^{\circ} \mathrm{C}$ overnight. After phosphate-buffered saline wash, slides were incubated with CY2conjugated streptavidin (Jackson ImmunoResearch, West Grove, PA) at room temperature for 1 hour. At least four $(\times 100)$-magnification immunofluorescent images were obtained for each mesenteric window using an upright fluorescent microscope (AX80; Olympus, Shinjuku, Tokyo, Japan). Images were then thresholded and quantified by Image $\mathrm{J}$ software [available for download from the National Institutes of Health (http://rsb.info.nih.gov/ij/)].

Hepatic Fibrosis Determination with Sirius Red Staining. Paraffin sections of liver were stained using Sirius red staining kit (Polysciences Inc., Warrington, PA). The percentage of Sirius redstained area was quantified by Image J. Briefly, the collagen stained in red was highlighted using color-thresholding function. The percentage of Sirius red-stained area was defined as measured thresholded area per image.

Western Blot. Microfuge tubes containing protein extraction solution was spun at $10,000 \mathrm{~g}$ for 10 minutes $\left(4^{\circ} \mathrm{C}\right)$. The supernatants were collected and protein concentrations were determined by a protein assay kit (Bio-Rad). One hundred micrograms of tissue lysates were mixed with $4 \times$ SDS loading dye to a final concentration of $2 \mu \mathrm{g} / \mu \mathrm{l}$. Twenty micrograms of protein samples were subjected to SDS-PAGE and transferred to a polyvinylidene fluoride membrane (Bio-Rad). The membranes were incubated with blocking solution (3\% bovine serum albumin) at room temperature for 1 hour, and then incubated with primary antibody at $4^{\circ} \mathrm{C}$ overnight: anti-VEGF rabbit monoclonal antibody (Millipore, Temecula, CA); anti-VEGFR2, p-VEGFR2, endothelial nitric oxide synthase (eNOS), p-eNOS, extracellular signal-regulated kinases (Erk), and p-Erk rabbit monoclonal antibody (Cell Signaling Technologies, Danvers, MA); anti- $\alpha$-smooth muscle actin ( $\alpha$-SMA) and tissue inhibitor of metalloproteinase (TIMP) 1 rabbit monoclonal antibody (Abcam, Cambridge, UK); matrix metalloproteinase (MMP) 13 and $\beta$-actin rabbit monoclonal antibody (GeneTex Inc., Irvine, CA). $\beta$-Actin served as loading control. The membrane was washed three times with Tris-buffered saline-Tween 20 (TBS-T) and then incubated with horseradishperoxidase (HRP)-conjugated secondary antibody at room temperature for 1 hour. Membranes were washed with TBS-T and developed with the enhanced chemiluminescence (Immobilon Western Chemiluminescent HRP Substrate; MilliporeSigma, Billerica, MA). The immunoreactive bands of specific proteins were detected by a digital luminescence imaging system (BioSpectrum 600 Imaging System; Ultra-Violet Products Ltd., Upland, CA). The signal intensity (integral volume) of the appropriate band was analyzed and quantified through Image J. 
TABLE 1

Hemodynamic parameters in nonviral cirrhotic rats treated with vehicle or NUCs

Values are expressed as mean \pm S.E.M. All $P>0.05$ among groups. Controls were treated with distilled water (vehicle).

\begin{tabular}{lcccc}
\hline & Control $(n=6)$ & Lamivudine $(n=5)$ & Entecavir $(n=5)$ & Tenofovir $(n=5)$ \\
\hline BW (g) & $368 \pm 20$ & $368 \pm 26$ & $381 \pm 17$ & $398 \pm 14$ \\
MAP (mm Hg) & $94 \pm 7$ & $92 \pm 3$ & $89 \pm 7$ & $100 \pm 3$ \\
HR (beats/min) & $254 \pm 22$ & $254 \pm 8$ & $246 \pm 26$ & $274 \pm 6$ \\
PP (mm Hg) & $13.5 \pm 0.8$ & $15.6 \pm 1.0$ & $13.7 \pm 2.7$ & $14.6 \pm 1.2$ \\
CI (ml/min per 100 g) & $46 \pm 3$ & $48 \pm 5$ & $37 \pm 4$ & $31 \pm 2$ \\
SVR (mm Hg/ml per minute per 100 g) & $2.1 \pm 0.3$ & $2.0 \pm 0.2$ & $2.5 \pm 0.5$ & $3.2 \pm 0.2$ \\
SMA flow (ml/min per 100 g) & $6.2 \pm 0.7$ & $7.0 \pm 1.0$ & $6.3 \pm 0.8$ & $6.1 \pm 0.4$ \\
SMA resistance (mm Hg/ml per minute & $13.8 \pm 2.0$ & $11.5 \pm 1.3$ & $12.5 \pm 1.7$ & $14.2 \pm 0.9$ \\
$\quad$ per 100 g) & & & & \\
Hepatic flow (portal part, ml/min per 100 g) & $8.2 \pm 1.1$ & $10.6 \pm 0.9$ & $8.3 \pm 1.2$ & $7.9 \pm 0.6$ \\
HVR (mm Hg/ml per minute per 100 g) & $1.8 \pm 0.26$ & $1.5 \pm 0.1$ & $1.8 \pm 0.3$ & $1.9 \pm 0.2$ \\
\hline
\end{tabular}

HVR, hepatic vascular resistance; $n$, number of rats; SVR, systemic vascular resistance.

Determination of Serum TNF- $\alpha$ Level. The plasma TNF- $\alpha$ levels were measured using a commercial enzyme-linked immunosorbent assay kit (R\&D Systems Inc., Minneapolis, MN) according to the manufacturer's instructions.

Drugs. Lamivudine was purchased from Glaxo Group Ltd. (Brentford, Middlesex, UK). Entecavir was purchased from Bristol-Myers Squibb (New York, NY). Tenofovir was purchased from Gilead Sciences (Foster City, CA). All the solutions were prepared freshly on the days of experiments.

Statistical Analysis. All results are expressed as mean \pm S.E.M. Statistical analyses were performed using an independent $t$ test, oneway analysis of variance, or repeated-measures analysis of variance as appropriate using SPSS 21 for Windows (SPSS Inc., Chicago, IL). Tukey honestly significant difference test was applied for post-hoc test. Results were considered statistically significant at a two-tailed $P$-value less than 0.05 .

\section{Results}

Systemic Effects of NUCs in Nonviral Cirrhotic Rats. As shown in Table 1, there was no significant difference in systemic and portal hemodynamic parameters, including MAP, HR, CI, PP, SVR and SMA resistance, among control, lamivudine, entecavir, and tenofovir groups. In short, the NUCs did not affect portal hypertension-related hemodynamic derangements in cirrhotic rats.
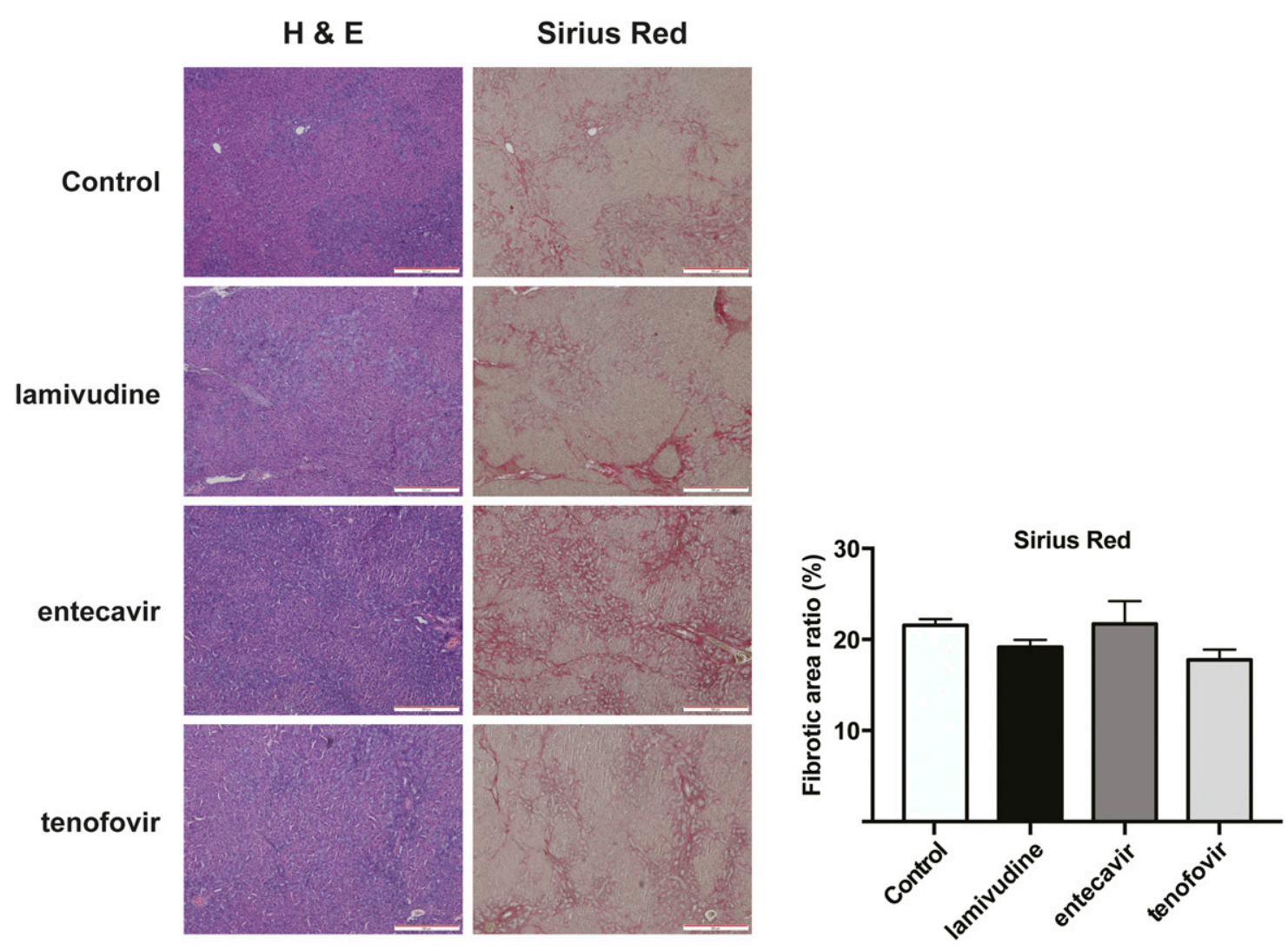

Fig. 1. Effects of NUCs on hepatic fibrosis area ratio in nonviral cirrhosis. Liver cirrhosis developed 4 weeks after CBDL. Sirius red stained the collagen fiber in red. Compared with distilled water (vehicle)-treated control group, lamivudine, entecavir, and tenofovir did not affect hepatic Sirius red staining area ratio. (Number: control, lamivudine, entecavir, tenofovir: 6, 5, 5, 5.) Scale bar, $500 \mu \mathrm{m}$. H \& E, hematoxylin and eosin. 

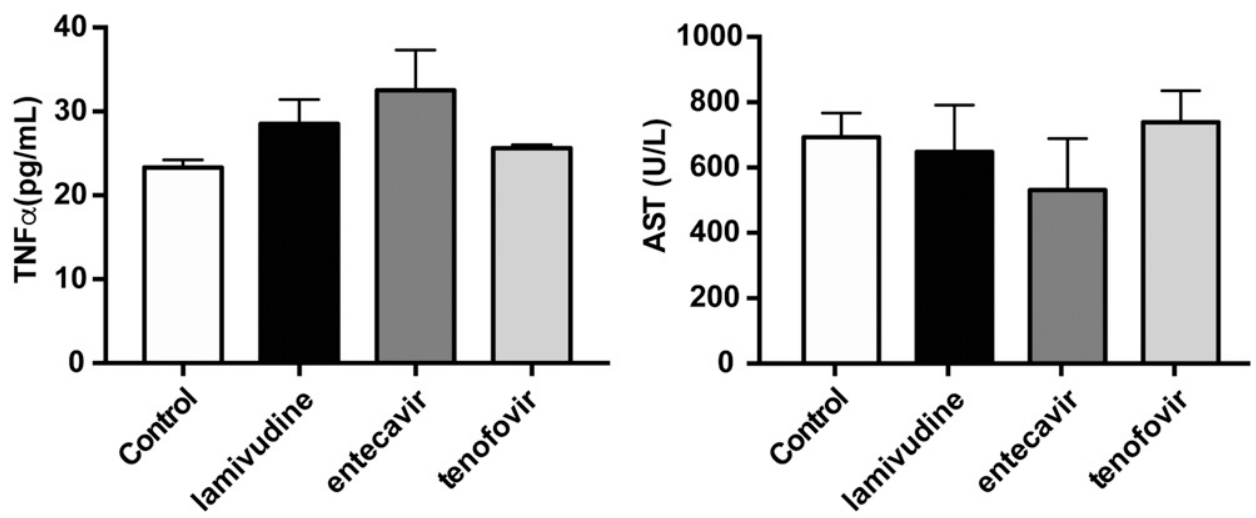

Fig. 2. Effects of NUCs on circulating hepatic inflammatory marker in nonviral cirrhosis. TNF- $\alpha$ plays an important role in hepatic inflammation. ALT, AST, and total bilirubin represents the severity of liver injury. All of the values were not
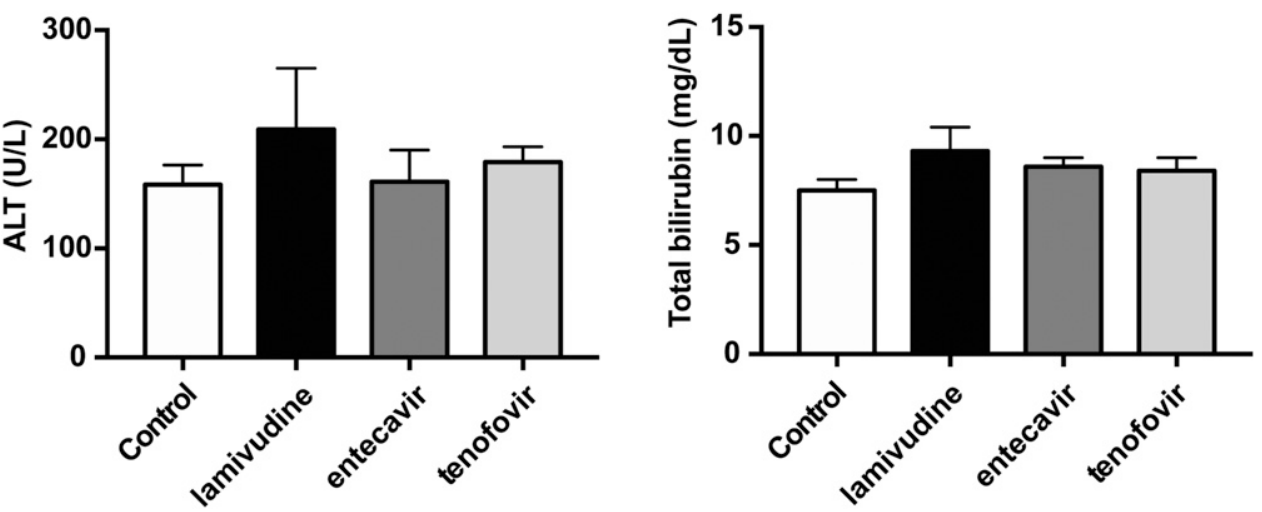
affected by NUCs. The results suggested NUCs did not influence nonviral inflammation and subsequent liver injury. ALT, alanine aminotransferase; AST, aspartate aminotransferase.

Effects of NUCs on Hepatic Fibrosis in Nonviral Cirrhotic Rats. The extent of hepatic fibrosis, evaluated by Sirius red staining, was not significantly different among NUCs and control groups (Fig. 1). The plasma TNF- $\alpha$ level, one of the cytokines that is linked to inflammation, was not significantly different among the four groups (Fig. 2; control, lamivudine, entecavir, tenofovir $(\rho \mathrm{g} / \mathrm{ml}): 23.4 \pm 0.85,28.5 \pm$ $2.86,32.5 \pm 4.83,25.6 \pm 0.42$ ). The plasma liver biochemistry parameters, including aspartate aminotransferase, alanine aminotransferase, and total bilirubin, were not significantly different among the four experimental groups as well (Fig. 2). The intrahepatic fibrogenesis-related protein expressions, including TIMP1, MMP13, and $\alpha$-smooth muscle actin $(\alpha$-SMA) were not significantly different among the four groups (Fig. 3).

Effects of NUCs on Angiogenesis in Nonviral Cirrhotic Rats. The mesenteric window vascular density was evaluated by CD31 immunofluorescence staining (Fig. 4). There was no significant difference in CD31-stained area ratio among the four groups (control, lamivudine, entecavir, tenofovir (\%): $11.03 \pm 1.06,10.51 \pm 2.10,8.39 \pm 0.92,7.46 \pm 1.39)$. Figure 5 presents the mesenteric proangiogenic protein expressions. Compared with the control group, VEGF receptor 2 (VEGFR2) phosphorylation was significantly downregulated by NUCs (control, lamivudine, entecavir, tenofovir: phosphoVEGFR2, $0.91 \pm 0.05,0.47 \pm 0.06,0.42 \pm 0.07,0.40 \pm 0.09$ control vs. lamivudine: $P=0.002$; control vs. entecavir: $P=$ 0.001; control vs. tenofovir: $P<0.001)$. The VEGF expression and Erk phosphorylation were upregulated in NUCs-treated groups, but the difference was not statistically significant (control, lamivudine, entecavir, tenofovir: VEGF, $0.62 \pm 0.16$, $1.10 \pm 0.08,1.02 \pm 0.24,1.00 \pm 0.28$; Erk phosphorylation,
$0.97 \pm 0.18,1.47 \pm 0.2,1.28 \pm 0.28,1.3 \pm 0.4)$. There was no significant difference on the phosphorylation of eNOS.

\section{Discussion}

In this study, the effects of lamivudine, entecavir, and tenofovir on nonviral cirrhosis were evaluated. Emerging data show that liver cirrhosis is no longer an irreversible status (Ismail and Pinzani, 2009). Human studies reveal that lamivudine, entecavir, and tenofovir suppress the progression of hepatic fibrosis and even reverse chronic hepatitis B-related liver cirrhosis (Kweon et al., 2001; Dienstag et al., 2003; Chang et al., 2010; Liaw, 2013; Marcellin et al., 2013). It is thought that the NUCs attenuate liver cirrhosis by suppressing HBVrelated liver damage. However, NUCs have been known to exert effects other than viral suppression, such as NO production (Zídek et al., 1999; Kostecká et al., 2012) and angiogenesis (Mizutani et al., 2015; Song et al., 2018). This study, therefore, assessed the therapeutic potential of NUCs on nonviral cirrhosis.

In this study, liver cirrhosis was induced by CBDL, a well established animal model with cholestatic liver injury. Indeed, there are several rat models with liver cirrhosis and portal hypertension, including $\mathrm{CCl}_{4}$ or thioacetamide administration and CBDL (Abraldes et al., 2006). For this study, the $\mathrm{CCl}_{4}$ and thioacetamide models may not have been feasible because both hepatotoxins share, at least partly, the same metabolic pathways as NUCs, especially that for the cytochrome P450s (Nekvindová et al., 2006; Xie et al., 2012); this could have interfered significantly with the results. On the other hand, the CBDL model induces liver injury by overt bile acid accumulation, which is considered far less an interference 

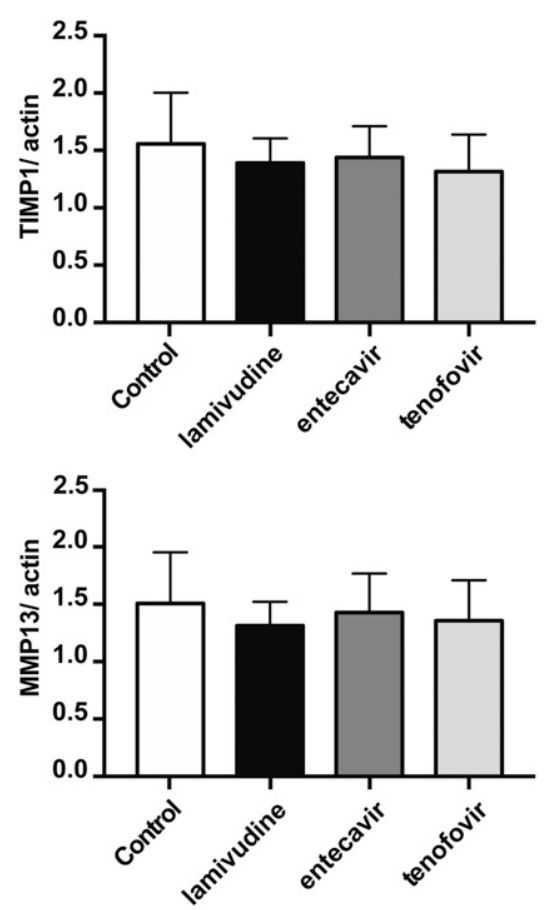
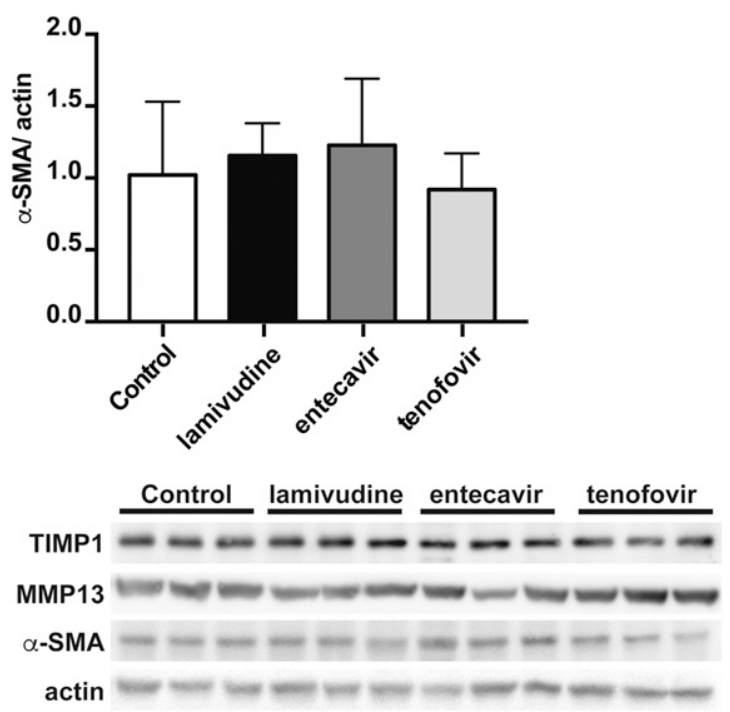

Fig. 3. Effects of NUCs on hepatic fibrogenic protein expression in nonviral cirrhosis. Liver injury activates hepatic stellate cells (HSCs), followed by collagen deposition. $\alpha$-SMA is the marker of HSCs activation. TIMP1 and MMP13 participate in hepatic fibrogenesis. None of the proteins were affected by chronic NUCs administration. (Number: control, lamivudine, entecavir, tenofovir: $5,5,5,5$.) with the NUCs metabolism (Jaeschke et al., 2002). As a result, we chose the CBDL model to test the effects of NUCs on liver cirrhosis and portal hypertension.

The overproduced collagen fiber in the liver is the cornerstone of liver cirrhosis. It is well recognized that TNF- $\alpha$ plays an important role in the process (Osawa et al., 2013). Interestingly, an in vitro study showed that tenofovir upregulated TNF- $\alpha$ expression in endometrial epithelial cells and fibroblasts (Biswas et al., 2014). Moreover, tenofovir enhanced the production of TNF- $\alpha$ in activated murine peritoneal macrophages in a dose-dependent manner (Zídek et al., 2000). However, in this study, the differences from control in the effect on plasma TNF- $\alpha$ levels were not statistically significant among the four groups. Consistently, the expression levels of activated hepatic stellate cells marker $\alpha$-SMA; TIMP1, the tissue inhibitor of metalloproteinase that promotes liver fibrosis (Yoshiji et al., 2000); and MMP13, the extracellular matrix enzyme participating in collagen degradation were comparable among the four groups. Overall, as indicated by hepatic Sirius red area ratio, NUCs did not affect the severity of liver fibrosis in nonviral cirrhosis.

Hyperdynamic circulation with increased splanchnic inflow contributes to portal hypertension, which results from excessive pathologic angiogenesis and splanchnic vasodilatation. The VEGF pathway is involved in the abnormal angiogenesis (Fernandez et al., 2004). Inhibition of VEGFR-2 decreases splanchnic hyperdynamic circulation and portosystemic collaterals in portal hypertensive rats (Fernandez et al., 2005). The effect of NUCs on angiogenesis inhibition has been noticed. Lamivudine attenuated laser-induced choroidal neovascularization in mice (Mizutani et al., 2015. A recent study also revealed that lamivudine and tenofovir inhibit VEGFR-2 phosphorylation in VEGF-stimulated human dermal microvascular endothelial cells (Song et al., 2018). In this study, compatible with the previous studies, the VEGFR-2 phosphorylation was significantly downregulated in NUCs-treated
Control

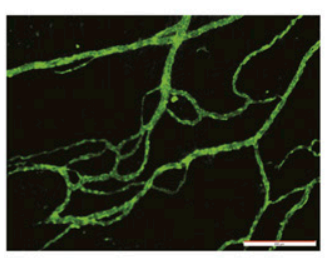

entecavir

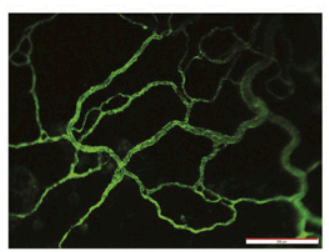

lamivudine

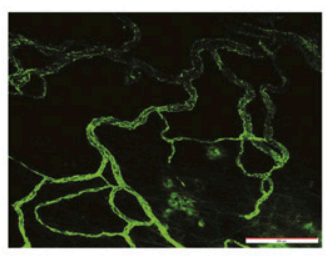

tenofovir

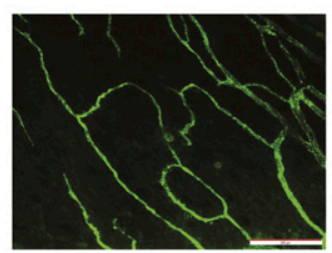

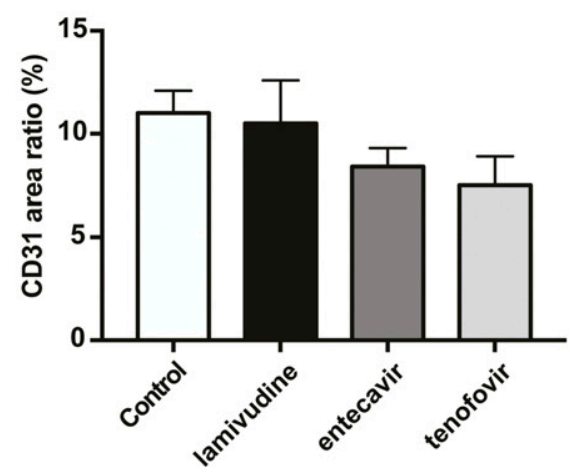

Fig. 4. Effects of NUCs on mesenteric angiogenesis in nonviral cirrhosis. Mesenteric angiogenesis was determined by vascular density. Chronic NUCs administration did not affect mesenteric vascular density. The representative figures are immunofluorescence staining of endothelial cell marker CD31 in mesenteric windows from CBDL-cirrhotic rats treated with vehicle or NUCs. (Number: control, lamivudine, entecavir, tenofovir: $6,5,5,5$.) Scale bar, $200 \mu \mathrm{m}$. 

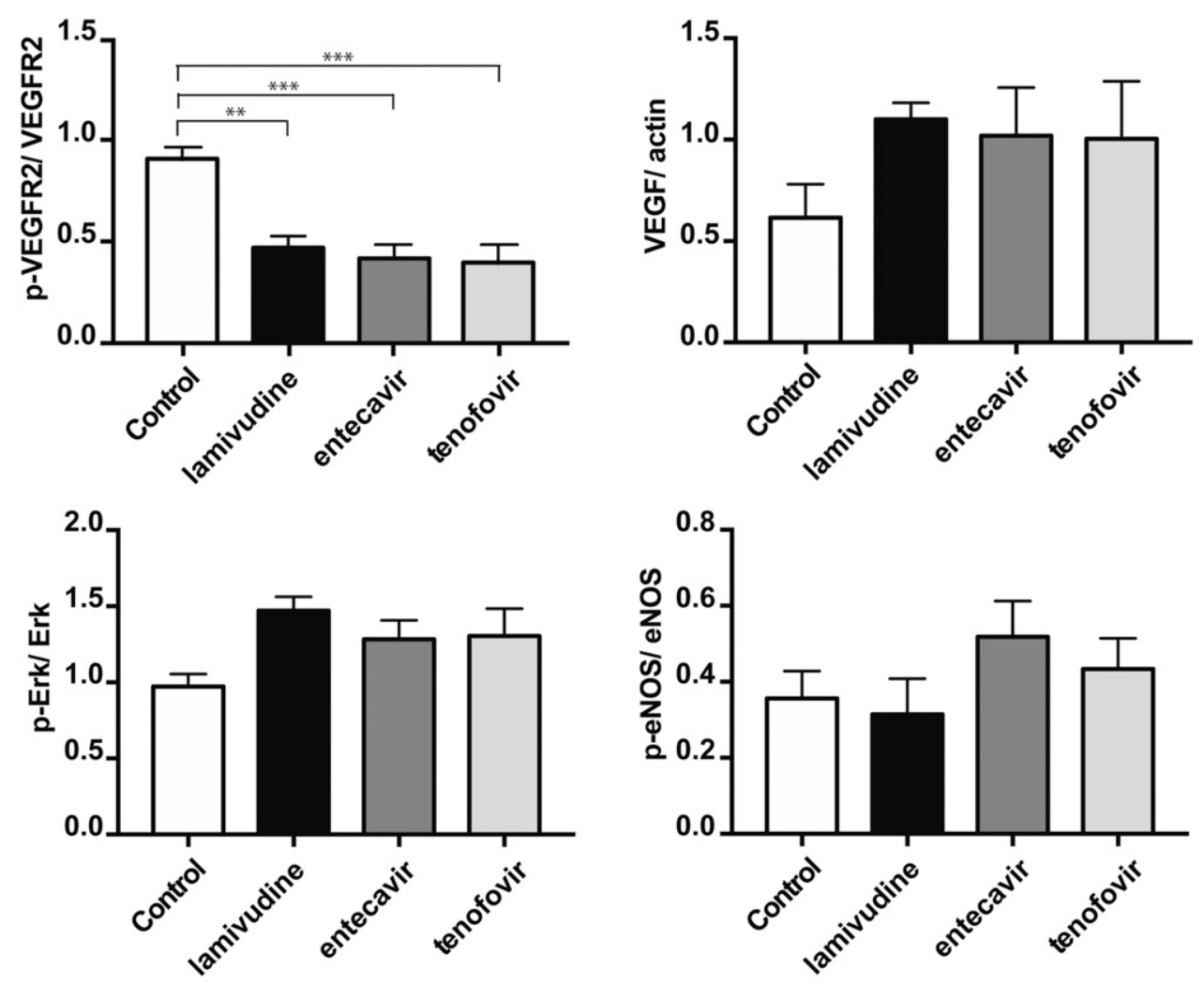

Fig. 5. Effects of NUCs on mesenteric protein expression in nonviral cirrhosis. The mesenteric protein expression of VEGFR2 phosphorylation, VEGF, Erk phosphorylation, and eNOS phosphorylation were evaluated by Western blotting. Compared with control group, NUCs administration significantly downregulated VEGFR2 phosphorylation. The expression level of VEGF and Erk phosphorylation were upregulated in NUCs treatment groups. eNOS phosphorylation was not affected by NUCs. (Number: control, lamivudine, entecavir, tenofovir: $5,5,5,5$.) $* * P \leq 0.01 ; * * * P \leq 0.001$ compared with

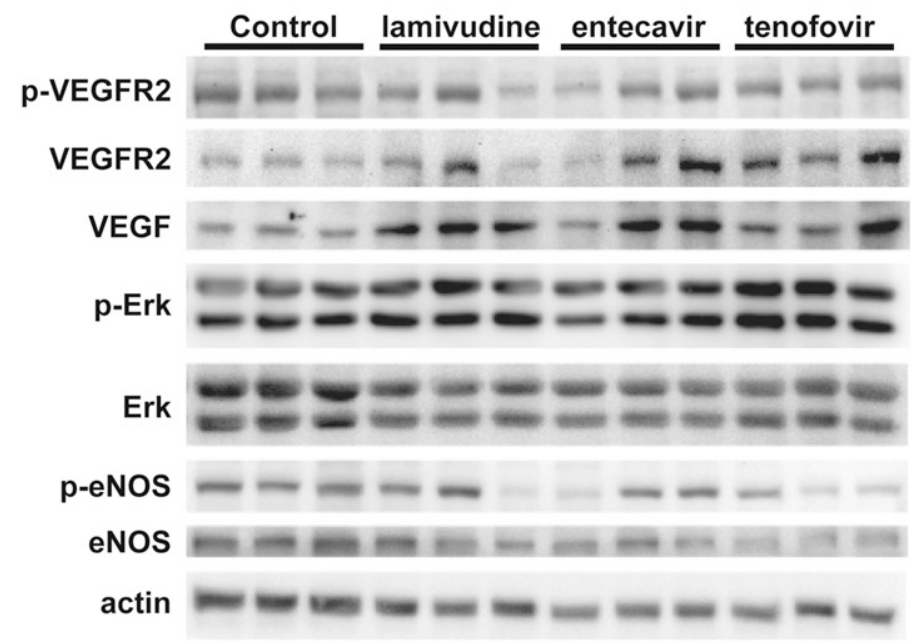
the control group.

cirrhotic rats. Interestingly, there was a trend toward upregulation in VEGF expression and Erk phosphorylation in NUCs-treated groups. The possible explanations could be a compensatory upregulation of VEGF and Erk in response to decreased VEGFR2 phosphorylation, different experimental setting, disease models, animal species, and/or targeted tissues. Nevertheless, the attenuated VEGFR2 activation indicated that the VEGF-signaling in this step was hampered by NUCs. It is thus inferred that the many angiogenesis and antiangiogenesis pathways that interplay during chronic NUCs treatment led to net results, as evident by mesenteric vascular density study, that were not statistically different between control and NUCs groups.

Excessive NO production during the progress of liver cirrhosis results in splanchnic vasodilatation (Iwakiri and
Groszmann, 2007) and angiogenesis (Sumanovski et al., 1999), which then exacerbate portal hypertension. Tenofovir has been found to stimulate NO production. An in vitro study showed that the nitrite (a stable metabolite of NO) concentration increased in rat peritoneal cells after 24 hours of tenofovir incubation (Kostecká et al., 2012). On the other hand, tenofovir applied subcutaneously for 16 days significantly increased serum and urine NO concentrations in female Lewis rats with arthritis (Zídek et al., 1999). In the present study, chronic NUCs treatment did not alter the hepatic eNOS phosphorylation and splanchnic blood inflow. These discrepant results may be attributable to different experimental designs, administration route and treatment duration of pharmacological agents, tissues under survey, and disease models. 
In conclusion, lamivudine, entecavir, and tenofovir did not affect hepatic fibrosis and portal hypertension-related derangements in nonviral cirrhosis. Despite downregulation of mesenteric VEGFR2 phosphorylation by NUCs, extrahepatic angiogenesis was not affected. Since NUCs exert a neutral effect on liver fibrosis and portal hypertension independent of HBV suppression, the clinical implication is that currently there is no evidence to support the use of NUCs beyond patients with chronic hepatitis B.

\section{Authorship Contributions}

Participated in research design: H.-C. Huang, F.-Y. Lee, Hsu, Y.-H.

Huang, Hou, S.-D. Lee.

Conducted experiments: Hsieh, Hsu.

Contributed new reagents or analytic tools:

Performed data analysis: Chang, Chuang.

Wrote or contributed to the writing of the manuscript: Hsieh, H.-C. Huang, Hsu.

\section{References}

Abraldes JG, Pasarín M, and García-Pagán JC (2006) Animal models of portal hypertension. World J Gastroenterol 12:6577-6584.

Albillos A, Colombato LA, and Groszmann RJ (1992) Vasodilatation and sodium retention in prehepatic portal hypertension. Gastroenterology 102:931-935.

Biswas N, Rodriguez-Garcia M, Shen Z, Crist SG, Bodwell JE, Fahey JV, and Wira CR (2014) Effects of tenofovir on cytokines and nucleotidases in HIV-1 target cells and the mucosal tissue environment in the female reproductive tract. Antimicrob Agents Chemother 58:6444-6453.

Cameron GR and Muzaffar Hasan S (1958) Disturbances of structure and function in the liver as the result of biliary obstruction. J Pathol Bacteriol 75:333-349.

Chang TT, Liaw YF, Wu SS, Schiff E, Han KH, Lai CL, Safadi R, Lee SS, Halota W, Goodman Z, et al. (2010) Long-term entecavir therapy results in the reversal of fibrosis/cirrhosis and continued histological improvement in patients with chronic hepatitis B. Hepatology 52:886-893.

Dienstag JL, Goldin RD, Heathcote EJ, Hann HW, Woessner M, Stephenson SL, Gardner S, Gray DF, and Schiff ER (2003) Histological outcome during long-term lamivudine therapy. Gastroenterology 124:105-117.

Fernandez M, Mejias M, Angermayr B, Garcia-Pagan JC, Rodés J, and Bosch J (2005) Inhibition of VEGF receptor-2 decreases the development of hyperdynamic splanchnic circulation and portal-systemic collateral vessels in portal hypertensive rats. $J$ Hepatol 43:98-103.

Fernandez M, Vizzutti F, Garcia-Pagan JC, Rodes J, and Bosch J (2004) Anti-VEGF receptor-2 monoclonal antibody prevents portal-systemic collateral vessel formation in portal hypertensive mice. Gastroenterology 126:886-894.

Huang HC, Wang SS, Hsin IF, Chang CC, Lee FY, Lin HC, Chuang CL, Lee JY, Hsieh HG, and Lee SD (2012) Cannabinoid receptor 2 agonist ameliorates mesenteric angiogenesis and portosystemic collaterals in cirrhotic rats. Hepatology 56:248-258.

Ismail MH and Pinzani M (2009) Reversal of liver fibrosis. Saudi J Gastroenterol 15 72-79.

Iwakiri Y and Groszmann RJ (2007) Vascular endothelial dysfunction in cirrhosis. $J$ Hepatol 46:927-934.

Jaeschke H, Gores G.J, Cederbaum AI, Hinson JA, Pessayre D, and Lemasters JJ (2002) Mechanisms of hepatotoxicity. Toxicol Sci 65:166-176.

Kostecká P, Holý A, Farghali H, Zídek Z, and Kmoníčková E (2012) Differential effects of acyclic nucleoside phosphonates on nitric oxide and cytokines in rat hepatocytes and macrophages. Int Immunopharmacol 12:342-349.

Kountouras J, Billing BH, and Scheuer PJ (1984) Prolonged bile duct obstruction: a new experimental model for cirrhosis in the rat. Br J Exp Pathol 65:305-311.

Kweon YO, Goodman ZD, Dienstag JL, Schiff ER, Brown NA, Burchardt E, Schoonhoven R, Brenner DA, and Fried MW (2001) Decreasing fibrogenesis: an immunohistochemical study of paired liver biopsies following lamivudine therapy for chronic hepatitis B [published correction appears in J Hepatol (2002) 36(5):714]. $J$ Hepatol 35:749-755.
Li FY, Hao HP, Hao K, Yan TT, and Wang GJ (2013) Effect of diammonium glycyrrhizinate on entecavir pharmacokinetics in rats. Chin J Nat Med 11:309-313.

Liaw YF (2013) Reversal of cirrhosis: an achievable goal of hepatitis B antiviral therapy. J Hepatol 59:880-881.

Lu CM, Hou ML, Lin LC, and Tsai TH (2014) Development of a microdialysis system to monitor lamivudine in blood and liver for the pharmacokinetic application in herbal drug interaction and the gene expression in rats. J Pharm Biomed Anal 96 231-240.

Marcellin P, Gane E, Buti M, Afdhal N, Sievert W, Jacobson IM, Washington MK, Germanidis G, Flaherty JF, Aguilar Schall R, et al. (2013) Regression of cirrhosis during treatment with tenofovir disoproxil fumarate for chronic hepatitis B: a 5-year open-label follow-up study. Lancet 381:468-475.

McConnell M and Iwakiri Y (2018) Biology of portal hypertension. Hepatol Int 12 (Suppl 1):11-23.

Mejias M, Garcia-Pras E, Tiani C, Miquel R, Bosch J, and Fernandez M (2009) Beneficial effects of sorafenib on splanchnic, intrahepatic, and portocollateral circulations in portal hypertensive and cirrhotic rats. Hepatology 49:1245-1256.

Mizutani T, Fowler BJ, Kim Y, Yasuma R, Krueger LA, Gelfand BD, and Ambati J (2015) Nucleoside reverse transcriptase inhibitors suppress laser-induced choroidal neovascularization in mice. Invest Ophthalmol Vis Sci 56:7122-7129.

Nekvindová J, Masek V, Veinlichová A, Anzenbacherová E, Anzenbacher P, Zídek Z and Holý A (2006) Inhibition of human liver microsomal cytochrome P450 activities by adefovir and tenofovir. Xenobiotica 36:1165-1177.

Nirogi R, Bhyrapuneni G, Kandikere V, Muddana N, Saralaya R, Komarneni P, Mudigonda K, and Mukkanti K (2012) Pharmacokinetic profiling of efavirenzemtricitabine-tenofovir fixed dose combination in pregnant and non-pregnant rats. Biopharm Drug Dispos 33:265-277.

Osawa Y, Hoshi M, Yasuda I, Saibara T, Moriwaki H, and Kozawa O (2013) Tumor necrosis factor- $\alpha$ promotes cholestasis-induced liver fibrosis in the mouse through tissue inhibitor of metalloproteinase-1 production in hepatic stellate cells. PLoS One 8: 65251.

Perry CM and Simpson D (2009) Tenofovir disoproxil fumarate: in chronic hepatitis B. Drugs 69:2245-2256.

Salerno JC, Ghosh DK, Razdan R, Helms KA, Brown CC, McMurry JL, Rye EA, and Chrestensen CA (2014) Endothelial nitric oxide synthase is regulated by ERK phosphorylation at Ser602. Biosci Rep 34:e00137.

Sims KA and Woodland AM (2006) Entecavir: a new nucleoside analog for the treatment of chronic hepatitis B infection. Pharmacotherapy 26:1745-1757.

Solà E, Lens S, Guevara M, Martín-Llahí M, Fagundes C, Pereira G, Pavesi M, Fernández J, González-Abraldes J, Escorsell A, et al. (2010) Hyponatremia in patients treated with terlipressin for severe gastrointestinal bleeding due to portal hypertension. Hepatology 52:1783-1790.

Song L, Ding S, Ge Z, Zhu X, Qiu C, Wang Y, Lai E, Yang W, Sun Y, Chow SA, et al. (2018) Nucleoside/nucleotide reverse transcriptase inhibitors attenuate angiogenesis and lymphangiogenesis by impairing receptor tyrosine kinases signalling in endothelial cells. Br J Pharmacol 175:1241-1259.

Sumanovski LT, Battegay E, Stumm M, van der Kooij M, and Sieber CC (1999) Increased angiogenesis in portal hypertensive rats: role of nitric oxide. Hepatology 29:1044-1049.

Xie Y, Wang G, Wang H, Yao X, Jiang S, Kang A, Zhou F, Xie T, and Hao H (2012) Cytochrome P450 dysregulations in thioacetamide-induced liver cirrhosis in rats and the counteracting effects of hepatoprotective agents. Drug Metab Dispos 40: 796-802.

Yoshiji H, Kuriyama S, Miyamoto Y, Thorgeirsson UP, Gomez DE, Kawata M, Yoshii J, Ikenaka Y, Noguchi R, Tsujinoue H, et al. (2000) Tissue inhibitor of metalloproteinases-1 promotes liver fibrosis development in a transgenic mouse model. Hepatology 32:1248-1254.

Zídek Z, Franková D, and Holý A (1999) Chemokines, nitric oxide and antiarthritic effects of 9-(2-phosphonomethoxyethyl)adenine (Adefovir). Eur J Pharmacol 376: 91-100.

Zídek Z, Franková D, and Holý A (2000) Macrophage activation by antiviral acyclic nucleoside phosphonates in dependence on priming immune stimuli. Int $J$ Immunopharmacol 22:1121-1129.

Address correspondence to: Dr. Shao-Jung Hsu, Division of Gastroenterology and Hepatology, Department of Medicine, Taipei Veterans General Hospital, No. 201, Sec. 2, Shih-Pai Road, Taipei 112, Taiwan. E-mail: sjhsu@ vghtpe.gov.tw 